Apidologie, 1982, 13 (2), 115-126.

\title{
ACTION DU CHLORAMPHÉNICOL SUR L'ÉVOLUTION DU SPECTRE PROTÉIQUE DE L'HÉMOLYMPHE D'ABEILLES NOURRIES SUR SIROPS DE SACCHAROSE ET DE TRÉHALOSE
}

\author{
Michel BOUNIAS (1) et M. R. J. MORGAN (2) \\ avec la collaboration technique de Mme C. JOLIET
}

\section{RÉSUMÉ}

Chez des abeilles naissantes nourries de sirops de saccharose pur $(2 \mathrm{M})$ ou de tréhalose ( $1 \mathrm{M})$, la protéinémie s'abaisse de manière continue, rapidement durant les 5 à 7 premiers jours, et plus lentement par la suite.

L'incorporation au régime alimentaire de $0,5 \mathrm{~g} / 1$ de Chloramphénicol $(1,6 \mathrm{mM})$ se traduit par une diminution significative des concentrations protéiques de l'hémolymphe, du $2^{\mathrm{e}}$ au $5^{\mathrm{e}}$ jour parès le début du traitement. Le maximum d'inhibition est observé au bout de 7 jours : son amplitude atteint $-60 \%$ avec la saccharose et $-10 \%$ avec le tréhalose. Enfin, le Chloramphénicol modifie certaines caractéristiques internes du spectre protéinique des abeilles élevées dans les conditions décrites.

\section{INTRODUCTION}

Les propriétés adaptatives des $\alpha$-glucosidases ont été étudiées depuis longtemps chez les vertébrés (Desnuelle, 1963).

L'un des principaux aspects de ce mécanisme est constitué par l'induction de la synthèse d'enzymes par leurs substrats, ces derniers pouvant soit provenir du métabolisme intermédiaire (ROBERT-BAUDOUY et al., 1974), soit être simplement introduits par le régime alimentaire (Firch et al., 1959; Desnuelle, 1973). Au cours d'une étude des propriétés d'inductibilité des $\alpha$-glucosidases de l'hémolymphe d'abeilles par des substrats naturels (Bounias et MorGaN, 1979), le rôle de la synthèse protéique a été exami-

(1) Laboratoire de Biochimie, I.N.R.A., Centre de Recherches d'Avignon B.P. 91, F 84140 Montfavet.

(2) Department of Biological Sciences, University of Lancaster. Great Britain. 
né au travers de son inhibition par le chloramphénicol (chloromycétine ou typhomycine). Cet antibiotique produit par une mycobactérie " actinomycétale " du genre streptomyces, est connu pour son action sur la synthèse des protéines des Bactéries gram (+) et gram (-), des Rickettsiées, des Spirochètes, de certains gros virus, ainsi que de Salmonella typhi (d'où l'une de ses dénominations usuelles). Mais de récents travaux ont montré que son action s'exerce également au niveau des protéines d'insectes (AsHour et al., 1978; Fragouli-ForNogeraKi et al., 1978) : l'objet du présent travail est donc d'étudier l'action du chloramphénicol sur l'évolution quantitative du spectre hémoprotéique d'abeilles élevées artificiellement sur sirops de saccharose (par référence au mode de nourrissement le plus fréquent en apiculture pratique) et de tréhalose en tant que substrat naturel dans l'hémolymphe de l'insecte (Evans et DETHIER, 1957).

\section{MATERIEL ET MÉTHODES}

Des abeilles naissantes (Apis mellifica, mellifica L.) sont placées au jour $\mathrm{J}$ par groupes de 60 dans des cagettes en matière plastique du type de celles utilisées pour les " hoarding tests " (KULINCEvic et RothENBUHLER, 1973). Les sirops de saccharose pur ( $2 \mathrm{M})$ et de tréhalose pur (1 M) sont présentés dans des tubes de $15 \mathrm{ml}$ perforés latéralement dans leur partie la plus basse. Deux autres lots d'abeilles reçoivent les mêmes substrats additionnés de chloramphénicol à la concentration de $0,5 \mathrm{~g} / 1(1,6 \mathrm{mM})$ ce qui correspond sensiblement à $10 \mu \mathrm{g}$ par abeille et par jour. Les prélèvements d'hémolymphe, les électrophorèses en gel de polyacrylamide $7,5 \%$ (en milieu Tampon TRIS-glycocolle $0,1 \mathrm{M} \mathrm{pH} 8,5$, à raison de $50 \mathrm{mn}$ sous $80 \mathrm{~V}$ puis $30 \mathrm{mn}$. sous $160 \mathrm{~V}$ ), le démoulage et les colorations au noir amido (amido-schwartz) sont opérés comme précédemment (Bounias, $1975 a$ et $b$ ).

L'analyse quantitative des différentes fractions protéiques est effectuée d'après l'enregistrement photométrique direct des gels. L'étalonnage quantitatif ainsi que la classification des poids molaires (P.M.) sont réalisés à partir des solutions étalons " HMW " et " LMW " (Pharmacia) ainsi que d'un groupe de sept protéines étalons traitées individuellement (PEI). Les calculs statistiques, moyennes, variances, corrélations, régressions, sont effectués au moyen d'une calculatrice SR 51 II (T - I). L'incertitude relative dans la détermination des teneurs protéiques, donnée par 3 répétitions des mesures pour un même échantillon, se situe comme suit (cas des témoins) : $x_{1}=564 ; 584 ; 600$ unités d'intégration. Moyenne : 582,7 ; écart-type : 18,04; intervalle de confiance au seuil $5 \%: 44,73$; soit : $\pm 7,7 \%$ de la moyenne. Les échantillons com prennent dix individus pour le prélèvement initial et sept pour tous les points suivants.

\section{RÉSULTATS}

\section{1. - Étalonnage pondéral et par classes de poids molaires}

La coloration par le noir amido donne, dans les limites de l'expérience, une relation linéaire entre la surface des pics d'enregistrements et les quantités correspondantes pour l'ensemble des protéines étalons de PM compris entre 20000 et 669000 . La figure 1 illustre les résultats obtenus avec l'albumine sérique bovine et avec l'ensemble 


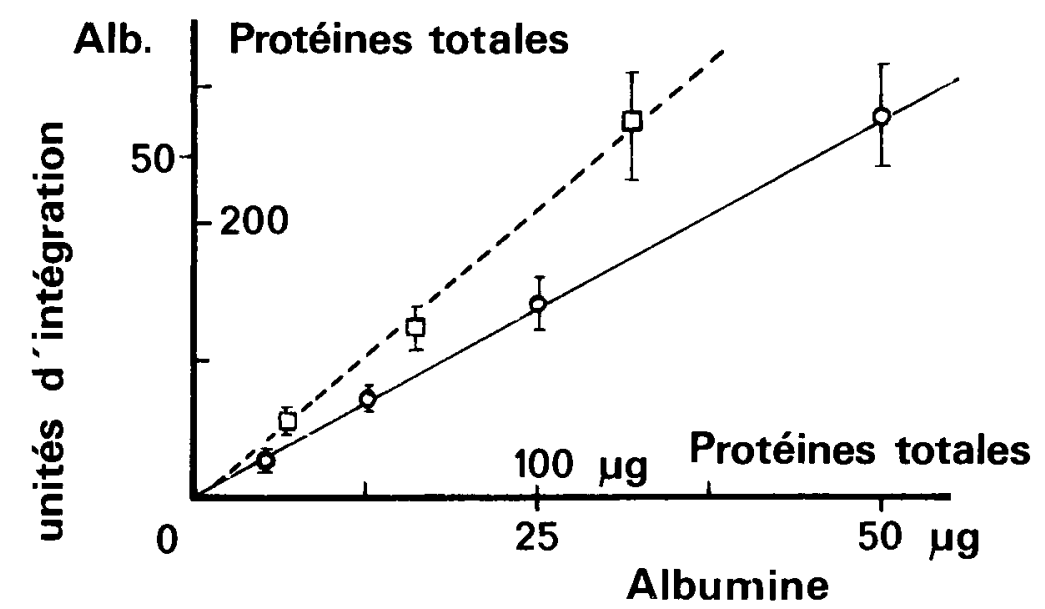

Fig. 1. - Courbes d'étalonnage de l'Albumine $(\mathrm{O}-\mathrm{O})$ et du groupe des protéines étalons " $H M W$ " (de P.M. compris entre 67000 et 669000$)(\square--\square$ ), après coloration au noir-amido et enregistrement photométrique direct.

Les intervalles de confiance sont indiqués au seuil $5 \%$ pour $\mathrm{N}=3$ mesures.

Fig. 1. - Albumin standardization curves $(\mathrm{O}-\mathrm{O})$ and standard protein groups * $H M W$, (of P.M. included between 67,000 and 669,000$)(\square--\square$ ) after coloration with black amido and direct photometric registration.

The confidence intervals are indicated at the $5 \%$ level for $\mathrm{N}=3$ measurements.

des cinq protéines de la série à hauts poids molaires ("HMW »). Les paramètres d'étalonnage correspondants s'établissent de la façon suivante :

Albumine : $\mathrm{N}=5$ points; corrélation $\rho=0,999$.

Pente : $p=2,200$ (ordonnée à l'origine : 0,42 ).

Protéines " $\mathrm{HMW}$ " : $\mathbf{N}=4$ points; corrélation $\rho=0,999$.

Pente : $p=2,162$ (ordonnée à l'origine : $-3,02$ ).

Les courbes peuvent être considérées comme linéaires et passant par l'origine.

Dans les conditions expérimentales observées pour l'électrophorèse, la mobilité relative $\left(\mathrm{M}_{R}\right)$ des fractions, exprimée par la distance parcourue relativement à la longueur du gel, est fonction inverse du logarithme naturel de leur poids molaire.

Les trois courbes d'étalonnage présentées sur la figure 2 montrent une grande concordance. Le calcul de régression de $\mathrm{L}_{\mathrm{N}}(\mathrm{PM})$ sur $\left(\mathrm{M}_{\mathrm{R}}\right)$ effectué sur l'ensemble des points $(\mathrm{N}=17)$ donne : $\rho=-0,968 ; a=13,030 ; b=-3,116$. Ceci correspond à des limites d'exclusion de 20200 à 448200 , dont les valeurs extrêmes se situent de 18500 à 590000 . 


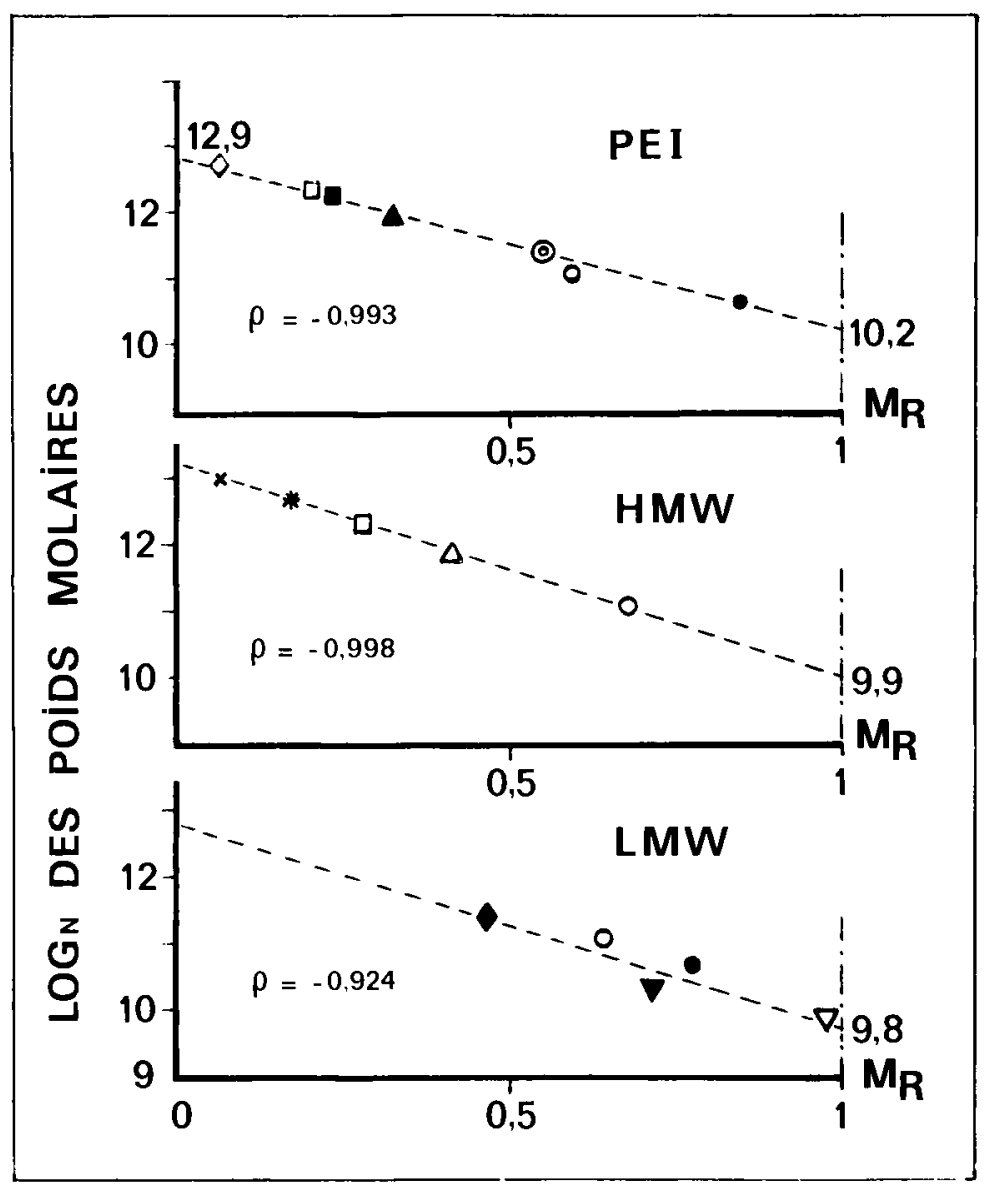

FIG. 2. - Courbes d'étalonnage du P.M. des protéines en fonction de leur mobilité (MR) exprimée relativement à la limite d'exclusion (longueur de gel : 65 à $70 \mathrm{~mm}$ ).

La courbe (PEI) correspond à des protéines analysées séparément (solutions individuelles) à raison de une par gel.

Les courbes HMW et LMW correspondent respectivement aux mélanges-étalons dits de "Haut" et de "Bas" poids molaires. Les limites d'exclusions sont précisées par le $L_{N}$ (P.M.).

Les protéines sont désignées par les symboles suivants : $(\diamond)=$ fibrinogène; $(\square)=$ catalase;

(ロ) $=\beta$-amylase; $(\mathbf{\Delta})=\gamma$-globuline; $(\mathrm{O})=$ albumine sérique bovine;

$(O)=$ albumine humaine; $(\bullet)=$ ovalbumine; $(x)=$ ferritine;

$(*)=$ thyroglobuline : sous-unité $\mathrm{n} / 2 ;(\Delta)=$ déshydrogénase lactique;

$(\boldsymbol{\nabla})=$ phosphorylase $b ;(\nabla)=$ anhydrase carbonique; $(\nabla)=$ inhibiteur de la trypsine.

FIG. 2. - Standardization curves of the P.M. of the proteins as a function of the mobility $\left(\mathrm{M}_{\mathrm{R}}\right)$ expressed in relation to the limit of exclusion (length of gel : 65 to $70 \mathrm{~mm}$ ).

The curves (PEI) correspond to proteins analyzed separately (individual solutions) at the rate of one per gel.

The curves HMW and LMW correspond respectively

to the mixtures-standards called " high" and "low " molecular weights.

The limits of exclusion are specified by the $\mathrm{L}_{\mathrm{N}}$ (P.M.).

The proteins are designated by the following symbols : $(\diamond)=$ fibrinogen; $(\square)=$ catalase;

$(\boldsymbol{\square})=\beta$-amylase; $(\boldsymbol{\Delta})=\gamma$-globulin; $(O)=$ bovine serum albumin; $(O)=$ human albumin;

$(\bullet)=$ ovalbumin; $(x)=$ ferritin; $(*)=$ thyroglobulin;

$(\Delta)=$ lactic dehydrogenase; sub-unit $\mathrm{n} / 2 ;(\diamond)=$ phosphorylase $b$;

$(\nabla)=$ carbonic anhydrase; $(\Delta)=$ trypsin inhibitor. 


\section{2. - Caractéristiques initiales du spectre protéique}

Le spectre protéique initial des abeilles naissantes utilisées au cours des expériences est comparable à celui observé cinq ans plus tôt au même stade (Bounias, 1975 a). Les douze fractions principales ont été retrouvées et leurs caractéristiques de concentrations, de mobilité et de classes de PM apparents sont exposées dans le tableau I. Par comparaison avec les échantillons analysés en 1974-1975, les lots de 1979 comportent seulement une inversion quantitative du rapport A I/A II en faveur de A II.

TABL. I. - Caractéristiques du spectre protéique initial des lots d'Abeilles étudiées. Les intervalles de confiance sont donnés au seuil $5 \%$ d'après $(\mathrm{N})$ mesures.

Les mobilités relatives $\left(\mathrm{M}_{\mathrm{R}}\right)$ sont exprimées relativement à la longueur du gel $(60$ à $65 \mathrm{~mm})$.

TABLE I. - Characteristics of the initial protein spectrum of the lots of honeybees studies. The confidence intervals are given at the $5 \%$ level after $(\mathrm{N})$ measurements.

The relative mobilities $\left(\mathrm{M}_{\mathrm{R}}\right)$ are expressed in relation to the length of the gel $(60$ to $65 \mathrm{~mm})$.

\begin{tabular}{c|c|c|c|c}
\hline \hline Fractions & $\begin{array}{c}\text { Concentrations } \\
\mu \mathrm{g} / 100 \mu 1 \\
(\mathrm{~N}=3) \\
\text { Fractions }\end{array}$ & $\begin{array}{c}\text { Concentrations } \\
\text { relatives \% } \\
\text { Relative } \\
\text { concentrations }\end{array}$ & $\mathbf{M}_{\mathrm{R}}$ & $\begin{array}{c}\text { Classes de poids molaire } \\
\text { apparent }(\mathrm{N}=\mathbf{4}) \\
\text { Classes of apparent } \\
\text { molar weights (N =4) }\end{array}$ \\
\hline A I & $129,0 \pm 8,2$ & 24,3 & 0,0175 & $427000 \pm 128000$ \\
A II & $191,4 \pm 32,2$ & 36,1 & 0,0775 & $356000 \pm 99000$ \\
B I & $18,8 \pm 10,6$ & 3,5 & 0,15 & $285000 \pm 72000$ \\
B II & $14,6 \pm 7,8$ & 2,7 & 0,18 & $260000 \pm 63000$ \\
C $_{1}$ & $33,6 \pm 4,6$ & 6,3 & 0,225 & $226000 \pm 52000$ \\
C $_{2}$ & $53,6 \pm 3,7$ & 10,1 & 0,275 & $195000 \pm 42000$ \\
$D_{2}$ & $35,2 \pm 9,4$ & 6,6 & 0,37 & $145000 \pm 27000$ \\
$E_{2}$ & $14,0 \pm 6,8$ & 2,6 & 0,49 & $101000 \pm 17000$ \\
$E_{3}$ & - & - & 0,57 & $80000 \pm 12000$ \\
$F_{1}$ & $1,8 \pm 1,4$ & 0,34 & 0,60 & $72000 \pm 11000$ \\
$F_{2}$ & - & - & 0,725 & $50000 \pm 8000$ \\
$F_{3}$ & $2,4 \pm 2,0$ & 0,45 & 0,795 & $40000 \pm 7000$ \\
\hline \hline
\end{tabular}

3. - Évolution comparée du spectre protéique en fonction du temps, en l'absence et en présence de chloramphénicol.

La figure 3 montre que la teneur protéique de l'hémolymphe diminue très rapidement au cours de la première semaine de nourrissement par le saccharose ainsi que par le tréhalose. La diminution est moins accentuée par la suite. En présence de chloramphénicol, la concentration protéique s'établit à un niveau constamment inférieur à celui des témoins à partir du deuxième jour avec la tréhalose et du cinquième jour avec le saccharose. Les différences restent significatives; elles sont maximales au bout de sept jours où elles atteignent $-60 \%$ dans le cas du saccharose et $-10 \%$ dans le cas du tréhalose. 


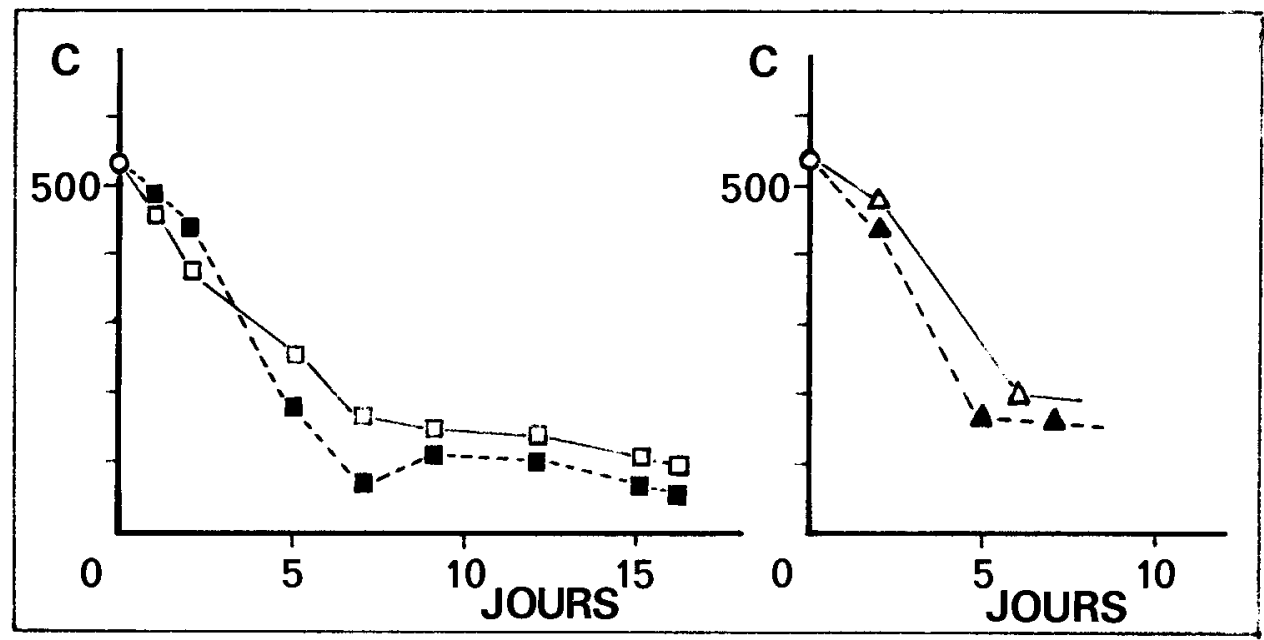

FIG. 3. - Variations de la concentration totale en protéines de l'hémolymphe (en $\mu \mathrm{g} / 100 \mu \mathrm{l})$ en fonction du temps et du régime alimentaire $:(\square)=$ saccharose seul;

$(\boldsymbol{\sigma})=$ saccharose + chloramphénicol; $(\Delta)=$ tréhalose seul;

$(\boldsymbol{\Delta})=$ tréhalose + chloramphénicol. $(\mathrm{O})=$ niveau initial.

FIG. 3. - Variations of the total concentration of hemolymph proteins (in $\mu \mathrm{g} / 100 \mu \mathrm{l}$ ) as a function of time and feeding regime: $(\square)=$ sucrose only; $(\mathbf{D})=$ sucrose + chloramphenicol; $(\Delta)=$ trehalose only; $(\Delta)=$ trehalose + chloramphenicol; $(O)=$ initial level.

L'évolution quantitative particulière des composants de chaque groupe, pour l'ensemble des quatre traitements, est précisée dans les tableaux II et III pour le saccharose et dans le tableau IV pour le tréhalose.

\section{DISCUSSION ET CONCLUSIONS}

Plusieurs points importants ressortent des données expérimentales obtenues au cours de cette étude.

\section{A. - Effets liés au nourrissement.}

La protéinémie des abeilles élevées sur milieux glucidiques purs subit une chute quantitative très rapide : ce phénomène peut donc s'analyser comme la conséquence d'une carence protéique alimentaire. D'autre part, les spectres protéiques respectifs des abeilles nourries sur saccharose et sur tréhalose présentent certaines différences perceptibles dès le deuxième jour (fig. 4) : dans le cas des abeilles recevant du tréhalose, la fraction $A$ II est beaucoup plus importante et la fraction $C_{1}$ dépasse quantitativement $\mathrm{C}_{2}$ : ces caractères évoquent le spectre des larves du cinquième stade (Bounias, 1975 a) dont l'hémolymphe est précisément très riche en tréhalose (BouNias, 1978). 


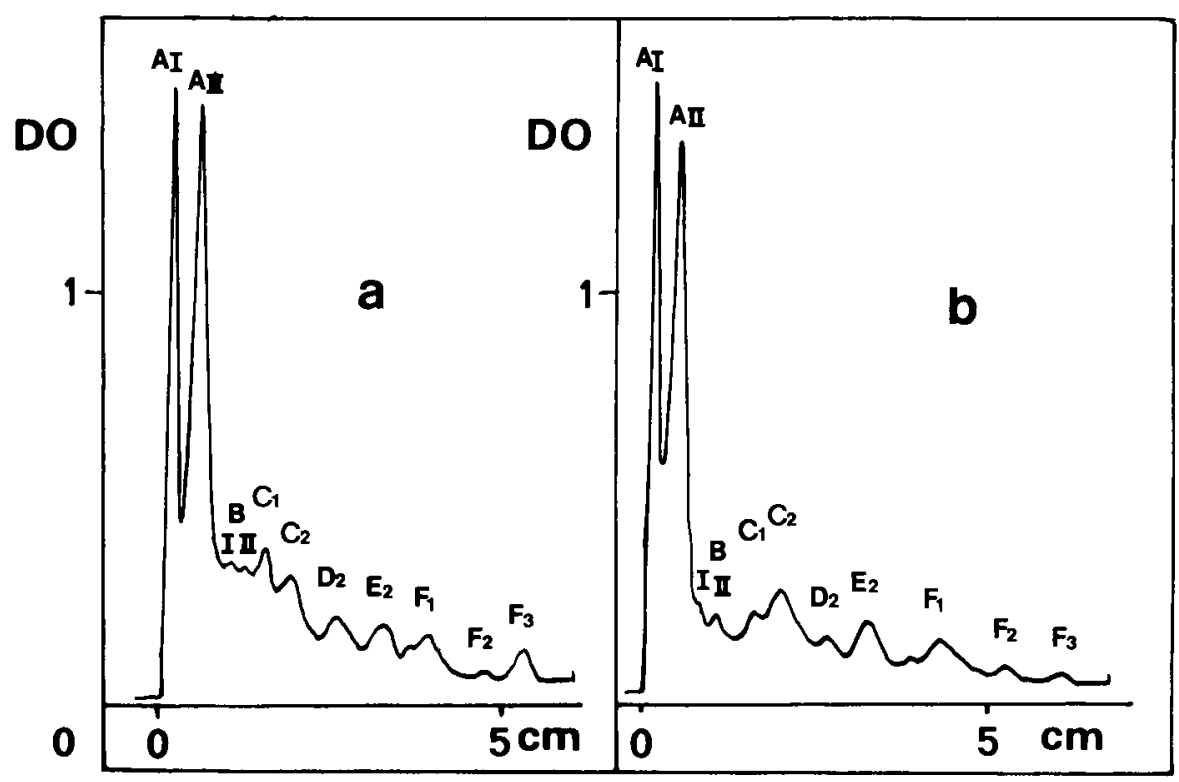

FIG. 4. - Comparaison des spectres protéiques de l'hémolymphe d'Abeilles nourries depuis 2 jours sur tréhalose (a) et sur saccharose (b).

FIG. 4. - Comparison of the protein spectra of the hemolymph of honeybees fed for 2 days on trehalose (a) and on sucrose (b).

Par ailleurs, l'évolution interne des spectres, en fonction du temps, s'effectue dans le sens d'un appauvrissement relatif de la fraction A II au profit des fractions du groupe $\mathrm{C}$ : or les groupes $\mathrm{A}$ et $\mathrm{C}$ sont ceux qui recèlent l'essentiel de l'activité $\alpha$ glucosidasique (Bounias, 1976) : du point de vue des poids molaires apparents, cela pourrait donc coïncider avec une dépolymérisation d'édifices de $P M \simeq 400000$ en sous unités de $P M \simeq 200000$. Cette modification interne du spectre protéique peut également apparaitre comme une conséquence du vieillissement des abeilles dont la composition hémoprotéique, exprimée en valeurs relatives, se rapproche fortement, en fonction du temps, de celle des abeilles butineuses (Bounias, 1975 ).

\section{B. - Effets liés à la présence de chloramphénicol.}

Le spectre protéique des abeilles recevant du tréhalose est quantitativement moins altéré par le chloramphénicol que celui des abeilles nourries sur saccharose. Cela pourrait suggérer l'hypothèse d'une "résistance " liée au fait que le tréhalose est le diholoside naturel de réserve de l'hémolymphe. Toutefois, la mortalité totale, au bout de 16 jours, atteint $21 \%$ sur saccharose et $50 \%$ sur trèhalose, ces chiffres étani ramenés respectivement à $2 \%$ et $45 \%$ en présence de chloramphénicol. Le tréhalose ne parait donc pas constituer une source nutritive capable de favoriser la survie des abeilles, tan- 
TABL. II. - Évolution du spectre protéique des Abeilles nourries sur saccharose pur, à partir du Jour $J+I$ (24 heures de traitement).

Les concentrations (C) en $\mu \mathrm{g} / 100 \mu \mathrm{l}$ sont suivies de la valeur relative entre parenthèses (\%). Coefficients de variation : $0,031(\mathrm{~N}=3)$.

TABLE. II. - Evolution of the protein spectrum of honeybees fed pure sucrose from day $J+I$ ( 24 hours after treatment).

The concentrations $(C)$ in $\mu \mathrm{g} / 100 \mu \mathrm{l}$ are followed by the relative value between parenthesis (\%). Coefficients of variation : $0.031(\mathrm{~N}=3)$.

\begin{tabular}{l|c|c|c|c|c|c|c|c|c}
\hline \hline & & $\mathbf{J}+1$ & $\mathrm{~J}+2$ & $\mathbf{J}+5$ & $\mathrm{~J}+7$ & $\mathrm{~J}+9$ & $\mathrm{~J}+12$ & $\mathbf{J}+15$ & $\mathbf{J}+16$ \\
\hline A I & $\mathrm{C}$ & 80,0 & 65,2 & 42,7 & 33,6 & 25,4 & 33,6 & 12,7 & 11,8 \\
& $\%$ & $(17,7)$ & $(17,5)$ & $(16,8)$ & $(21,3)$ & $(17,5)$ & $(26,0)$ & $(13,2)$ & $(13,7)$ \\
$\mathrm{A}$ II & $\mathrm{C}$ & 176,4 & 71,6 & 64,5 & 26,4 & 16,4 & 9,1 & 10,9 & 10,9 \\
& $\%$ & $(38,9)$ & $(19,2)$ & $(25,3)$ & $(16,7)$ & $(11,2)$ & $(7,0)$ & $(11,3)$ & $(12,6)$ \\
$\mathrm{B} \mathrm{I}$ & $\mathrm{C}$ & 10,9 & 11,1 & 9,1 & 6,4 & 6,4 & 5,5 & 5,4 & 3,7 \\
& $\%$ & $(2,4)$ & $(3,0)$ & $(3,6)$ & $(4,0)$ & $(4,4)$ & $(4,2)$ & $(5,6)$ & $(4,2)$ \\
B II & $\mathrm{C}$ & 9,1 & 12,7 & 10,9 & 5,4 & 7,2 & 4,6 & 4,5 & 3,7 \\
& $\%$ & $(2,0)$ & $(3,4)$ & $(4,3)$ & $(3,5)$ & $(5,0)$ & $(3,5)$ & $(4,7)$ & $(4,2)$ \\
$\mathrm{C}_{1}$ & $\mathrm{C}$ & 18,2 & 15,9 & 25,4 & 10,0 & 9,1 & 7,3 & 7,2 & 6,4 \\
& $\%$ & $(4,0)$ & $(4,3)$ & $(10,0)$ & $(6,3)$ & $(6,2)$ & $(5,6)$ & $(7,5)$ & $(7,4)$ \\
$\mathrm{C}_{2}$ & $\mathrm{C}$ & 52,7 & 58,9 & 63,6 & 36,4 & 47,3 & 36,4 & 28,2 & 21,8 \\
& $\%$ & $(11,6)$ & $(15,8)$ & $(25,0)$ & $(23,0)$ & $(32,5)$ & $(28,2)$ & $(29,2)$ & $(25,2)$ \\
$\mathrm{D}_{2}$ & $\mathrm{C}$ & 16,4 & 14,3 & 9,1 & 9,0 & 6,4 & 9,1 & 9,1 & 9,1 \\
& $\%$ & $(3,6)$ & $(3,8)$ & $(3,6)$ & $(5,7)$ & $(4,4)$ & $(7,0)$ & $(9,4)$ & $(10,5)$ \\
$\mathrm{E}_{2}$ & $\mathrm{C}$ & 30,9 & 41,4 & 13,6 & 5,4 & 10,0 & 5,4 & 3,6 & 1,8 \\
& $\%$ & $(6,8)$ & $(11,1)$ & $(5,4)$ & $(3,5)$ & $(6,9)$ & $(4,2)$ & $(3,8)$ & $(2,1)$ \\
$\mathrm{F}_{\mathbf{l}}$ & $\mathrm{C}$ & 25,4 & 38,2 & 6,4 & 2,8 & 2,7 & 1,9 & 1,8 & 1,4 \\
& $\%$ & $(5,6)$ & $(10,2)$ & $(2,5)$ & $(1,7)$ & $(1,8)$ & $(1,4)$ & $(1,8)$ & $(1,6)$ \\
$\mathrm{F}_{2}$ & $\mathrm{C}$ & 3,6 & 4,8 & 0,9 & 0,9 & 0,9 & - & 0,9 & 0,9 \\
& $\%$ & $(0,8)$ & $(1,3)$ & $(0,4)$ & $(0,6)$ & $(0,6)$ & - & $(0,9)$ & $(1,0)$ \\
$\mathrm{F}_{3}$ & $\mathrm{C}$ & 0,9 & 1,6 & - & - & - & - & - & - \\
& $\%$ & $(0,2)$ & $(4,3)$ & - & - & - & - & - & - \\
\hline \hline
\end{tabular}

dis que l'adjonction de chloramphénicol atténue, au contraire, le taux de mortalité. Il n'est pas à exclure que ce dernier point soit à relier à l'activité antibiotique de l'effecteur. Enfin, la présence de chloramphénicol modifie certaines caractéristiques du spectre protéique de l'hémolymphe des abeilles recevant du tréhalose : en particulier, la fraction $C_{1}$ reste plus élevée que $C_{2}$ alors que l'inverse est observé dans tous les autres cas.

\section{C. - Intérêt pour l'étude des mécanismes d'induction.}

Les recherches effectuées au niveau des $\alpha$-glucosidases, en relation avec le nourrissement, ont montré une augmentation d'activité dont la maximum se situe au bout de sept jours dans le cas du saccharose, et de cinq jours dans le cas du tréhalose (Bounias et MoRgaN, 1979). L'action inhibitrice du chloramphénicol coïncide donc parfaite- 
TABL. III. - Évolution du spectre protéique des Abeilles nourries sur saccharose + chloramphénicol à partir de $(J+1)=24$ heures après le début du traitement.

$\mathrm{C}=$ concentrations en $\mu \mathrm{g} / 100 \mu \mathrm{l}$. Les valeurs relatives (\%) sont indiquées entre parenthèses.

TABLE III. - Evolution of the protein spectrum of honeybees fed on sucrose plus chloramphenicol starting from $(J+I)=24$ hours after the onset of treatment.

$\mathrm{C}=$ concentrations in $\mu \mathrm{g} / 100 \mu \mathrm{l}$. The relative values (\%) are indicated between parenthesis.

\begin{tabular}{l|c|c|c|c|c|c|c|c|c}
\hline & & $\mathrm{J}+1$ & $\mathrm{~J}+2$ & $\mathrm{~J}+5$ & $\mathrm{~J}+7$ & $\mathrm{~J}+9$ & $\mathrm{~J}+12$ & $\mathrm{~J}+15$ & $\mathrm{~J}+16$ \\
\hline A I & $\mathrm{C}$ & 129,0 & 74,5 & 48,2 & 17,3 & 17,3 & 18,1 & 10,0 & 9,1 \\
& $\%$ & $(26,5)$ & $(17,1)$ & $(27,3)$ & $(27,5)$ & $(14,0)$ & $(17,8)$ & $(14,1)$ & $(16,6)$ \\
A II & $\mathrm{C}$ & 178,5 & 86,4 & 41,8 & 10,9 & 26,4 & 10,0 & 10,0 & 7,3 \\
& $\%$ & $(36,6)$ & $(20,0)$ & $(23,7)$ & $(17,4)$ & $(21,3)$ & $(9,8)$ & $(14,1)$ & $(13,3)$ \\
B I & $\mathrm{C}$ & 11,2 & 7,3 & 5,4 & 4,5 & 5,4 & 4,5 & 4,5 & 2,7 \\
& $\%$ & $(2,3)$ & $(1,7)$ & $(2,8)$ & $(7,2)$ & $(4,4)$ & $(4,5)$ & $(6,4)$ & $(5,0)$ \\
B II & $\mathrm{C}$ & 9,7 & 8,2 & 4,5 & 3,6 & 5,4 & 2,7 & 3,6 & 2,7 \\
& $\%$ & $(2,0)$ & $(1,9)$ & $(2,4)$ & $(5,8)$ & $(4,4)$ & $(2,7)$ & $(5,1)$ & $(5,0)$ \\
C I & $\mathrm{C}$ & 8,2 & 13,6 & 9,1 & 4,6 & 7,3 & 4,5 & 7,3 & 6,4 \\
& $\%$ & $(1,7)$ & $(2,5)$ & $(5,1)$ & $(7,2)$ & $(5,9)$ & $(4,5)$ & $(10,2)$ & $(11,7)$ \\
$\mathrm{C}_{2}$ & $\mathrm{C}$ & 19,5 & 27,3 & 33,6 & 16,4 & 33,6 & 37,3 & 20,9 & 19,1 \\
& $\%$ & $(4,0)$ & $(6,2)$ & $(19,0)$ & $(26,0)$ & $(27,2)$ & $(36,6)$ & $(29,4)$ & $(35,0)$ \\
$\mathrm{D}_{2}$ & $\mathrm{C}$ & 6,8 & 11,8 & 10,0 & 0,9 & 7,3 & 37 & 3,6 & 2,7 \\
& $\%$ & $(1,4)$ & $(2,7)$ & $(5,7)$ & $(1,4)$ & $(5,8)$ & $(3,6)$ & $(5,1)$ & $(5,0)$ \\
$\mathrm{E}_{2}$ & $\mathrm{C}$ & 12,0 & 16,4 & 10,0 & 0,9 & 4,5 & 2,7 & 1,8 & 0,9 \\
& $\%$ & $(2,5)$ & $(3,7)$ & $(5,7)$ & $(1,4)$ & $(3,7)$ & $(2,7)$ & $(2,6)$ & $(1,7)$ \\
$\mathrm{F}_{1}$ & $\mathrm{C}$ & 10,5 & 19,1 & 4,5 & - & 1,8 & 1,8 & - & - \\
& $\%$ & $(2,1)$ & $(4,4)$ & $(2,6)$ & - & $(1,5)$ & $(1,8)$ & - & - \\
$\mathrm{F}_{2}$ & $\mathrm{C}$ & 1,5 & 1,8 & 0,9 & - & 0,9 & - & - & - \\
& $\%$ & $(0,3)$ & $(0,4)$ & $(0,5)$ & - & $(0,7)$ & - & - & - \\
\hline \hline
\end{tabular}

ment avec l'évolution de l'induction enzymatique, et constitue donc dans ce cas précis un instrument particulièrement favorable à l'étude de la relation de ce mécanisme adaptatif avec la synthèse protéique.

\section{REMERCIEMENTS} 1981.

Les auteurs tiennent à rendre hommage à l'excellente collaboration de Mme JoLIET, décédée en juillet

Les abeilles proviennent du rucher de la station d'Apiculture, confié aux soins de M. R. PARIs. Le Dr Morgan a bénéficié de subventions " Leverhulme Trust Fund ", " I.C.I. Plant Protection ", " Shell International Petroleum ", et d'une bourse de la " Royal Society ". 
TAB. IV. - Évolution du spectre protéique des Abeilles nourries sur tréhalose pur ou additionné de chloramphénicol.

Les concentrations $\mathrm{C}$ sont exprimées en $\mu \mathrm{g} / 100 \mu \mathrm{l}$ et les valeurs relatives (\%) notées entre parenthèses. Coefficients de variation : $0,031(\mathrm{~N}=3)$.

TABLE IV. - Evolution of the protein spectrum of honeybees fed on pure trehalose or on trehalose supplemented with chloramphenicol.

The concentrations (C) are expressed in $\mu \mathrm{g} / 100 \mu \mathrm{l}$, and the relative values (\%) noted between parentheses. Coefficients of variation : $0.031(\mathrm{~N}=3)$.

\begin{tabular}{|c|c|c|c|c|c|c|}
\hline \multicolumn{4}{|c|}{$\begin{array}{l}\text { Tréhalose pur } \\
\text { Pure trehalose }\end{array}$} & \multicolumn{3}{|c|}{$\begin{array}{c}\text { Tréhalose + chloramphénicol } \\
\text { Trehalose + chloramphenicol }\end{array}$} \\
\hline & & $\mathbf{J}+2$ & $\mathrm{~J}+5 / \mathrm{J}+7$ & $\mathbf{J}+2$ & $J+5$ & $\mathrm{~J}+7$ \\
\hline A I & $\begin{array}{c}\mathrm{C} \\
\%\end{array}$ & $\begin{array}{c}57,3 \\
(12,2)\end{array}$ & $\begin{array}{c}18,2 \\
(18,3)\end{array}$ & $\begin{array}{c}49,1 \\
(11,0)\end{array}$ & $\begin{array}{c}30,0 \\
(16,7)\end{array}$ & $\begin{array}{c}32,7 \\
(18,4)\end{array}$ \\
\hline A II & $\begin{array}{l}\mathrm{C} \\
\%\end{array}$ & $\begin{array}{l}212,7 \\
(45,5)\end{array}$ & $\begin{array}{c}70,9 \\
(35,6)\end{array}$ & $\begin{array}{l}209,0 \\
(46,5)\end{array}$ & $\begin{array}{c}47,3 \\
(26,2)\end{array}$ & $\begin{array}{c}40,0 \\
(22,4)\end{array}$ \\
\hline B I & $\begin{array}{l}\mathrm{C} \\
\%\end{array}$ & $\begin{array}{l}19,1 \\
(4,1)\end{array}$ & $\begin{array}{c}9,1 \\
(4,6)\end{array}$ & $\begin{array}{l}20,0 \\
(4,5)\end{array}$ & $\begin{array}{c}9,1 \\
(5,0)\end{array}$ & $\begin{array}{l}10,0 \\
(5,6)\end{array}$ \\
\hline B II & $\begin{array}{l}\mathrm{C} \\
\%\end{array}$ & $\begin{array}{l}21,8 \\
(4,7)\end{array}$ & $\begin{array}{l}10,0 \\
(5,0)\end{array}$ & $\begin{array}{l}14,5 \\
(3,2)\end{array}$ & $\begin{array}{c}9,1 \\
(5,0)\end{array}$ & $\begin{array}{c}6,4 \\
(3,6)\end{array}$ \\
\hline $\mathrm{C}_{1}$ & $\begin{array}{l}\mathrm{C} \\
\%\end{array}$ & $\begin{array}{l}45,4 \\
(9,7)\end{array}$ & $\begin{array}{l}16,4 \\
(8,2)\end{array}$ & $\begin{array}{c}72,7 \\
(16,1)\end{array}$ & $\begin{array}{c}52,7 \\
(29,2)\end{array}$ & $\begin{array}{c}43,6 \\
(24,5)\end{array}$ \\
\hline $\mathrm{C}_{2}$ & $\begin{array}{l}\mathrm{C} \\
\%\end{array}$ & $\begin{array}{l}34,5 \\
(7,4)\end{array}$ & $\begin{array}{c}29,1 \\
(14,6)\end{array}$ & $\begin{array}{l}27,3 \\
(6,1)\end{array}$ & $\begin{array}{l}10,9 \\
(6,0)\end{array}$ & $\begin{array}{l}16,4 \\
(9,2)\end{array}$ \\
\hline $\mathrm{D}_{2}$ & $\begin{array}{l}\mathrm{C} \\
\%\end{array}$ & $\begin{array}{l}27,3 \\
(5,8)\end{array}$ & $\begin{array}{l}10,0 \\
(5,0)\end{array}$ & $\begin{array}{l}18,2 \\
(4,0)\end{array}$ & $\begin{array}{l}10,0 \\
(5,5)\end{array}$ & $\begin{array}{c}9,1 \\
(5,1)\end{array}$ \\
\hline $\mathrm{E}_{2}$ & $\begin{array}{l}\mathrm{C} \\
\%\end{array}$ & $\begin{array}{l}25,4 \\
(5,4)\end{array}$ & $\begin{array}{c}6,4 \\
(3,2)\end{array}$ & $\begin{array}{l}14,5 \\
(3,2)\end{array}$ & $\begin{array}{c}3,6 \\
(2,0)\end{array}$ & $\begin{array}{c}9,1 \\
(5,1)\end{array}$ \\
\hline $\mathrm{E}_{3}$ & $\begin{array}{l}\mathrm{C} \\
\%\end{array}$ & $\begin{array}{c}9,1 \\
(1,9)\end{array}$ & $\begin{array}{c}1,8 \\
(0,9)\end{array}$ & $\begin{array}{c}4,5 \\
(1,0)\end{array}$ & $\begin{array}{c}0,9 \\
(0,5)\end{array}$ & $\begin{array}{c}0,9 \\
(0,5)\end{array}$ \\
\hline$F_{1}$ & $\begin{array}{l}\mathrm{C} \\
\%\end{array}$ & $\begin{array}{l}14,2 \\
(3,1)\end{array}$ & $\begin{array}{c}2,7 \\
(1,4)\end{array}$ & $\begin{array}{r}9,1 \\
(2,0)\end{array}$ & $\begin{array}{c}0,9 \\
(0,5)\end{array}$ & $\begin{array}{c}1,8 \\
(1,0)\end{array}$ \\
\hline$F_{2}$ & $\begin{array}{l}\mathrm{C} \\
\%\end{array}$ & $\begin{array}{c}3,6 \\
(0,8)\end{array}$ & $\begin{array}{c}9,1 \\
(0,5)\end{array}$ & $\begin{array}{r}0,9 \\
(0,2)\end{array}$ & - & - \\
\hline $\mathbf{F}_{3}$ & $\begin{array}{l}\mathrm{C} \\
\%\end{array}$ & $\begin{array}{c}7,3 \\
(1,6)\end{array}$ & $\begin{array}{l}0,0 \\
-\end{array}$ & $\begin{array}{r}1,8 \\
(0,4)\end{array}$ & $\begin{array}{l}0,0 \\
-\end{array}$ & $\begin{array}{c}1,8 \\
(1,0)\end{array}$ \\
\hline
\end{tabular}

Reçu pour publication en mai 1981. Eingegangen im Mai 1981.

\section{SUMMARY}

ACTION OF CHLORAMPHENICOL ON THE MODIFICATION OF PROTEIN SPECTRUM OF HONEYBEES REARED ON SUCROSE AND TREHALOSE SYRUPS

The study of the action of chloramphenicol on the quantitative and qualitative hemoprotein spectrum of emerging honeybees reared on sucrose and trehalose syrups was made in view of investigations on the nature of the induction of hemolymph alpha-glucosidases by their natural substrates.

The hemolymph protein concentration of honeybees receiving either sucrose $(2 \mathrm{M})$ or trehalose $(1 \mathrm{M})$ decreases rapidly during the first 5 to 7 days and then tends to become stabilized. This reduction in proteins is interpreted as an effect of nutritional protein deficiency. 
The addition of chloramphenicol at the rate of $0.5 \mathrm{~g} / 1(1.6 \mathrm{mM})$ to each of the two regimes resulted in a reduction of the protein contents in relation to the treatments without the inhibitor. In the case of trehalose, the decrease is significant from the 2nd day and reaches a maximum of $-10 \%$ at the end of 5 days; with sucrose, the effect is significant at the end of 5 days and presents a maximum of $-60 \%$ on the 7 th day.

Certain internal modifications of the protein spectrum appear to be connected to the nutrition (relative increase from the trehalose of certain fractions of apparent high molar weight); the action of chloramphenicol tends to accentuate this phenomenon in the case of nutrition with trehalose. Finally, the addition of chloramphenicol reduces the rate of mortality from $21 \%$ to $2 \%$ in the case of sucrose, and from 50 to $45 \%$ in the case of trehalose.

\section{ZUSAMMENFASSUNG}

\section{WIRKUNG DES CHLORAMPHENICOLS AUF DIE ENTWICKLUNG DES PROTEINSPEKTRUMS DER HAEMOLYMPHE DER BIENEN, DIE MIT SACCHAROSE - UND TREHALOSELÖSUNGEN GEFÜTTERT WORDEN WAREN}

In Zusammenhang mit Forschungen über die Art der Induktion der $\gamma$-Glucosidase der Haemolymphe durch ihre natürlichen Substrate wurde eine Untersuchung der quantitativen und qualitativen Wirkung des Chloramphenicols auf das Proteinspektrum des Blutes von schlüpfenden Bienen durchgeführt, die künstlich mit Saccharose - und Trehalosesyrup aufgezogen worden sind.

In den Bienengruppen, die entweder reine Saccharose $(2 \mathrm{M})$ oder reine Trehalose (1 M) erhielten, sank die Konzentration der Haemolymphproteine im Verlaufe der ersten 5-7 Tage rapide ab, dann strebte sie einer Stabilisierung zu. Dieser Proteinabfall wird als Folge des Proteinmangels in der Nahrung interpretiert. Die Zugabe von Chloramphenicol in einer Konzentration von 0,5/1 (1,6 mM) zu jedem der beiden Ansätze kommt in einer Senkung des Proteingehaltes im Vergleich zu den Ansätzen ohne diesen Hemmfaktor zum Ausdruck. Im Fall der Trehalose wird die Senkung ab dem 2. Tag signifikant und sie erreicht ihren Höhepunkt von - $10 \%$ nach Ablauf von 5 Tagen. Bei Saccharose ist der Effekt am Ende des 5. Tages signifikant und er erreicht sein Maximum von $-60 \%$ am 7. Tag.

Gewisse interne Modifikationen des Proteinspektrums schienen mit der Ernährung in Verbindung zu stehen (relative Vermehrung bestimmter hochmolarer Fraktionen durch die Trehalose). Die Chloramphenicol-Wirkung verstärkt dieses Phänomen im Fall der Ernährung mit Trehalose. Schliesslich verringert der Zusatz von Chloramphenicol die Mortalitätsrate im Falle der Saccharose von $21 \%$ auf $2 \%$ und im Fall der Trehalose von 50 auf $45 \%$.

\section{RÉFÉRENCES BIBLIOGRAPHIQUES}

Ashour B., Tribe M., WhitTaker P., 1980. - The effect of chloramphenicol succinate, ethidium bromide and cycloheximide on mortality and mitochondrial protein synthesis of adult blowflies. J. Cell. Science (in press).

Bounias M., 1975 a. - Les protéines de l'hémolymphe chez l'abeille Apis mellifica, mellifica L. I. - Larves, Nymphes et Adultes naissantes. Apidologie, 6, 207-218.

Bounias M., $1975 \mathrm{~b}$. - Les protéines de l'hémolymphe chez l'abeille Apis mellifica, mellifica L. II. Adultes butineuses et hivernantes. Apidologie, 6, 219-232.

Bounias M., 1976. - Paramètres cinétiques de l'activité $\alpha$-glucosidasique de l'hémolymphe chez l'abeille ouvrière (Apis mellifica, mellifica L.). Apidologie, 7, 263-275. 
Bounias M., 1978. - Intra-glycemic relation occuring during the development of honeybee workers. Comp. Biochem. Physiol., 61 A, 13-18.

Bounias M., Morgan M. R. J., 1979. - L'inductibilité de l'activité des $\alpha$-glucosidases de l'hémolymphe chez l'abeille. C.R. Ann. Trav. Lab. Biochimie, 8, 17-27.

Desnuelle P., 1963. - Quelques exemples d'adaptation enzymatique chez les mammifères. Rev. Franç. Études Clin. et Biol., 8, 494-506.

Desnuelle P., 1973. - Adaptation of the Biosynthesis of Pancreatic Enzymes to Nutritional and Hormonal Factors. Proc. 2nd int. Coll. " Automatisation and Prospective Biology ", Pont-à-Mousson 1972 (Karger, Basel, 1973), 22-26.

Evans D. R., DethieR V. G., 1957. - The regulation of taste threshold for sugars in the blowfly. J. Insect. Physiol., 1, 3-17.

Fitch W. M., Hill R., Chaikoff I. L., 1959. - Hepatic glycolytic enzyme activities in the alloxan diabetic rat : response to glucose and fructose feeding. J. Biol. Chem., 234, 2811.

Fragoult-Fournogeraki M. E., Fragoulis E. G., Sekeris C. E., 1978. - Protein synthesis by polysomes from the epidermis of blowfly larvae : dependence of formation of dopa-decarboxylase on developmental stage. Insect Biochem., 8, 435-441.

KULINCEvic J. M., Rothenguhler C., 1973. - Laboratory and field measurement of hoarding behaviour in the honeybee. J. Apic. Res., 12, 179-182.

Robert-Baudouy J. M., Portalier R. C., Stoeber F. C., 1974. - Régulation du métabolisme des hexuronates chez Escherichia coli $\mathrm{K} 12$. Modalités de l'induction des enzymes du systèmes hexuronate. Eur. J. Biochem., 43, 1-15. 